\title{
The Current Recommended Drugs and Strategies for the Treatment of Coronavirus Disease (COVID-19)
}

This article was published in the following Dove Press journal:

Therapeutics and Clinical Risk Management

\section{Mojgan Sheikhpour $\mathbb{D}^{1,2}$}

'Department of Mycobacteriology and Pulmonary Research, Pasteur Institute of Iran, Tehran, Iran; ${ }^{2}$ Microbiology Research Center, Pasteur Institute of Iran, Tehran, Iran
Correspondence: Mojgan Sheikhpour

Tel +98-91229697/2

$\mathrm{Fax}+982164112313$

Email mshaikhpoor@gmail.com
Background: The coronavirus 2019 (COVID-19) has been known as a pandemic disease by the World Health Organization (WHO) worldwide. The drugs currently used for treatment of COVID-19 are often selected and tested based on their effectiveness in other diseases such as influenza and AIDS and their major identified targets are viral protease, host cell produced protease, viral RNA polymerase, and the interaction site of viral protein with host cell receptors. Until now, there are no approved therapeutic drugs for definitive treatment of this dangerous disease.

Methods: In this article, all of the documentary information, such as clinical trials, original research and reviews, government's database, and treatment guidelines, were reviewed critically and comprehensively. Moreover, it was attempted to present the most common and effective drugs and strategies, to suggest the possible treatment way of COVID19 by focusing on the body's defense mechanism against pathogens.

Results: Antiviral drugs and immune-modulatory agents with the traditional medicines using the natural compound are usual accessible treatments. Accordingly, they have better beneficence due to the large existence studies, long time follow-ups, proximity to the natural system, and the normal physiological routine of the pathogen and host interactions. Besides, the serotonergic and dopaminergic pathways are considered as attractive targets to treat human immune, infectious, and cancerous diseases. Fluoxetine, as a host-targeted small molecule with immunomodulatory action, may be known as effective drug for treatment and prevention of COVID19 disease, in combination with antiviral drugs and natural compounds.

Conclusion: Co-administration of fluoxetine in the treatment of COVID19 could be considered due to the possibility of its interaction with ACE2 receptors, immune-modulatory function, and a proper immune response at the right time. Fluoxetine plays a beneficial role in reducing stress due to fear of infecting by COVID19 or worsening the disease and psychological support for the affected patients.

Keywords: COVID-19, clinical trials, therapeutic drugs, immunomodulatory, fluoxetine

\section{Introduction}

In January 2020, World Health Organization (WHO) discovered a new coronavirus disease emerged in Wuhan, Hubei Province. Correspondingly, this disease, caused by a novel SARS-CoV-2, has been named as the novel coronavirus, 2019-nCoV or COVID-19. ${ }^{1}$ The WHO characterized the COVID-19 as a pandemic disease, which has been already spread in many countries that resulted in numerous infections and unfortunate human deaths worldwide. ${ }^{2}$ So, the COVID-19 epidemic is unique 
because of its high spread, incidence, and mortality rates all around the world. The outbreak of this virus is the third highly pathogenic coronavirus, which has infected human being in the 20th and 21st centuries. ${ }^{3,4}$ In this regard, several trials have been listed in the reported clinical trials, research works or government databases such as the remdesivir, favipiravir, lopinavir, ritonavir, oseltamivir, methylprednisolone, bevacizumab, human immunoglobulin, interferons, chloroquine, hydroxychloroquine, arbidol, etc. Besides, alternative medicine such as traditional Chinese medicines (TCM) or a combination of some of these trials together have been used to get probably successful treatment. $^{5}$

Even after performing several types of research on the COVID-19, there are still no approved vaccines or therapeutic drugs for its treatment. So, there is a need to develop some effective agents and vaccines for successful treatment and future epidemics prevention of this dangerous and fatal disease. Comprehensive and intensive clinical trials using the traditional Chinese medicine and western medicine, are ongoing in China; however, due to the low quality, small sample size, and long duration of these studies, still no certain treatment way of COVID-19 has been achieved for a long time in the future. ${ }^{6}$ However, for a long time, clinical use should be ethically approved by the WHO, and safety and high-quality clinical trial data are also needed. ${ }^{7}$

The big family of coronaviruses is diverse in both phenotypical and genetical aspects. They have enveloped viruses containing single-stranded positive-sense RNA with the ability to cause infection in birds, mammals, and humans. The genome of the virus encodes the structural and non-structural proteins, which is $27-32 \mathrm{~kb}$. Accordingly, the membrane (M), envelope (E), nucleocapsid $(\mathrm{N})$, and spike $(\mathrm{S})$ are the structural proteins, which play a key role in the virus entry and replication in the host cell (Figures 1 and 2). ${ }^{8,9}$

Human coronaviruses have access to the host cells via some specific receptors, which are present on the host cells. It was indicated that the pathogenesis of a coronavirus strongly depends on the interaction of the $\mathrm{S}$ protein with its receptor. Several studies have shown that blocking of S1 subunit and protease inhibition can prevent the SARS-CoV -2 entrance into target cell, since host receptor binding is mediated by the $\mathrm{N}$ terminal domain-NTD of spike protein $\mathrm{S} 1$ subunit. Also, some researchers suggested that $\mathrm{S} 1$ subunit and host proteases play potential therapeutic roles in the treatment of COVID-19 (Figure 3). ${ }^{10}$ In this regard, it was shown that Zoonotic $\beta$-coronaviruses have a high binding affinity towards angiotensin converting enzyme 2(ACE2), which serves as an operative receptor for viral entry to the host cell. ACE2 have also been reported as cellular receptors used by $\beta$-coronaviruses MERS-CoV and bat coronavirus. Accordingly, a high propensity associated with ACE2 receptor and S1 domain of spike protein has been shown. ${ }^{11}$

Due to many produced humanity losses caused by this virus in a short time worldwide, many doctors and scientists are trying to find a way to prevent and also to fight this disease. In a short time, many studies and reports have been published, which one could face lots of scattered information. Moreover, there are some review studies in terms of the introduction of drugs or methods, more or less, on their usage; but there is still no review on the definite and possible benefits and challenges of these drugs or methods. Therefore, in this review article, as far as the documentary information is concerned, it was tried to present the methods and drugs that may have been proposed and used for the prevention and treatment of COVID19. This comprehensive and discussing review study has been performed referring to the effect mechanisms, benefits, and challenges of each drug or method. In this article, based on the valuable studies, a possible drug and treatment strategy that may be considered in clinical trials is been proposed.

\section{Search Methodology}

In this article, all the documentary information such as clinical trials and original research and reviews obtained from some international databases such as Google Scholar, PubMed, Scopus, Web of Science, and Science Direct were searched for the following MeSH words: notably, COVID19 with each one of the following words: clinical trial, drug, treatment, therapy, alternative medicine, nano-medicine, antiviral, anti-inflammatory, stem cell, plasma, and TCM. Moreover, the government's databases and treatment guidelines were also reviewed critically and comprehensively. Correspondingly, it was attempted to present the most common and effective drugs and strategies, in order to suggest the possible treatment way of COVID19 by focusing on the body's defense mechanism against pathogens.

\section{Antiviral Drugs and Anti-Inflammatory Agents}

Based on several types of research and clinical trials in different countries, the lopinavir/ritonavir (LPV/r) can be recommended from the early stage of COVID-19 
A
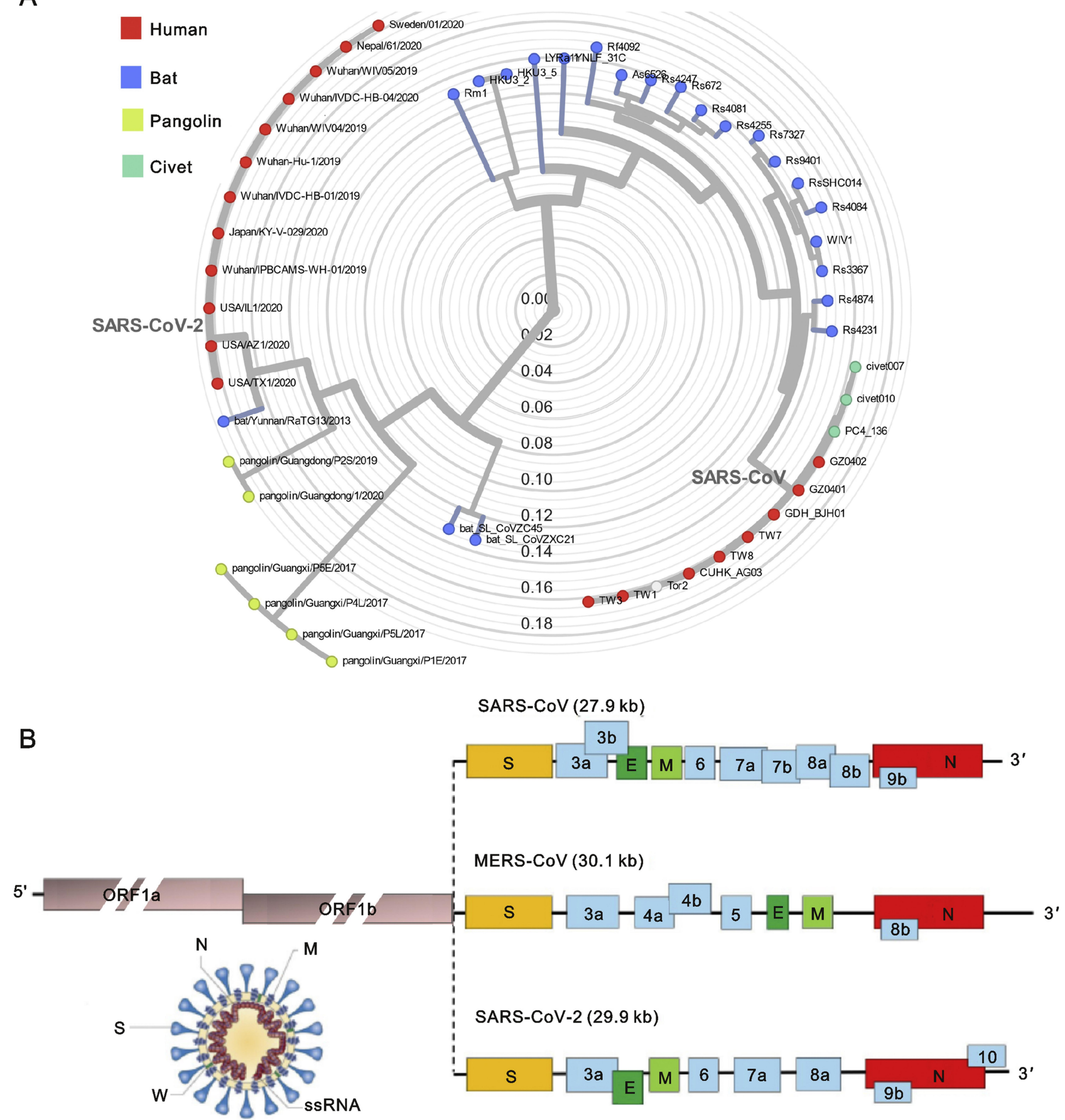

Figure I (A) The phylogenetic tree of SARS-like coronaviruses complete genome sequences. (B) The complete genome sequences of SARS-CoV, MERS-CoV, and SARS-CoV-2. Notes: Reprinted from Journal of Pharmaceutical Analysis, Vol I0/edition number 2, Li X, Geng M, Peng Y, Meng L, Lu S, Molecular immune pathogenesis and diagnosis of COVID-19, Pages No.102-108, Copyright (2020), with permission from Elsevier. ${ }^{8}$

pneumonia either to the patients suffering from underlying diseases or to elderly patients. Notably, LPV/r reduces the viral loads and improves the clinical symptoms throughout the treatment. LPV/r previously optimized for the treatment and prevention of HIV/AIDS viral infection and also shown in vitro and in vivo protease inhibitory effect on SARS-CoV2. ${ }^{12}$ However, more evidence from some wellcontrolled clinical trials is needed to demonstrate the clinical efficacy of LPV/r. ${ }^{13,14}$ The data obtained from the registered clinical trials for COVID-19 up to March 7, 


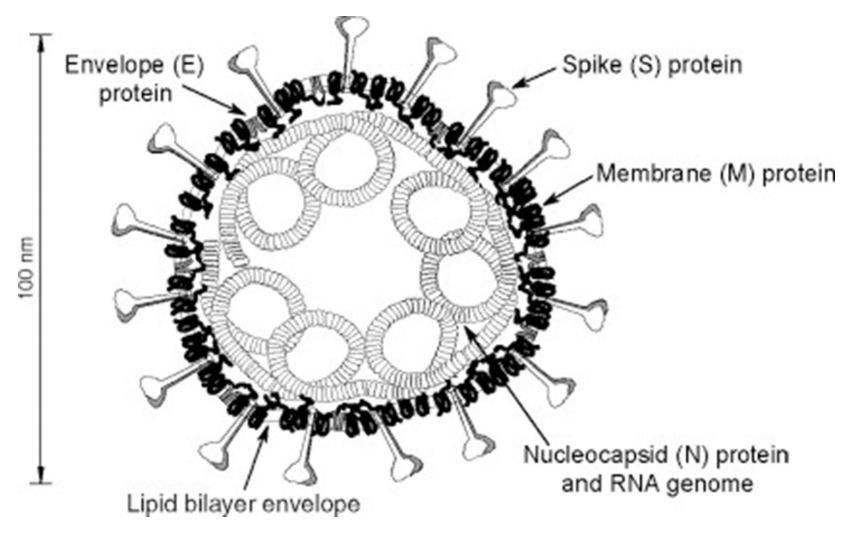

Figure $2 \mathrm{~A}$ vision of coronavirus with the minimal set of structural proteins. Notes: Reprinted from Advances in Virus Research, Vol 66, Masters PS, The molecular biology of coronaviruses, Pages No.193-292, Copyright (2006), with permission from Elsevier.'

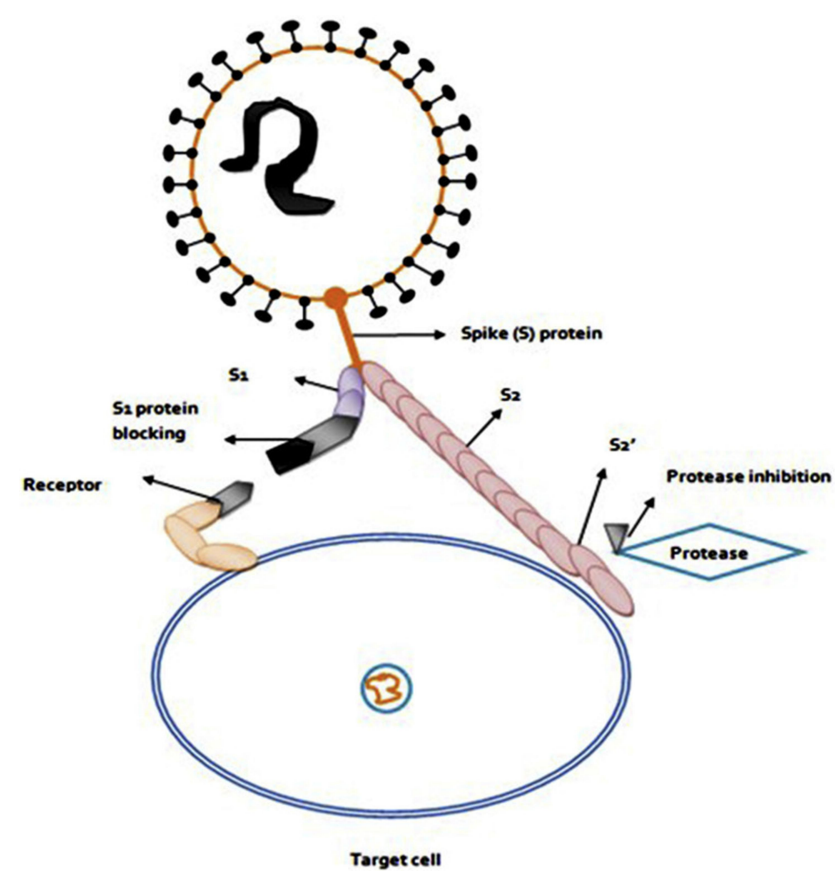

Figure 3 Blocking of SI Subunit and protease inhibition prevents the SARS-CoV-2 entry into target cell. Therefore SI subunit and host proteases are potential therapeutic ways for the treatment of COVID-19.

Notes: Reprinted from Archives of Medical Research, Vol 66, Arafah A, Ali S, Yatoo AM, Ali MN, Rehman MU, SI subunit and host proteases as potential therapeutic avenues for the treatment of COVID-19, In press, Copyright (2020), with permission from Elsevier. ${ }^{10}$

2020, showed that the stem cells therapy and LPV/r were the most frequently evaluated therapies, used in 23 and 15 trials, respectively. While the remdesivir was associated with the highest median number of the planned inclusions per trial (400, IQR 394-453) only for 5 trials. ${ }^{15-17}$ Zhang et $\mathrm{al}^{18}$ considered 125 clinical trials on the treatment of COVID-19, registered up to February 18, 2020, which were conducted by pharmaceutical companies, government, institutions, physicians, and scientists. Accordingly, the results showed that $33.3 \%$ of all the clinical trials used anti-viral agents, $14.7 \%$ antiinflammation or immune modulators, $33.3 \%$ herbs or traditional Chinese medicine (TCM), 9.3\% cell-based therapy, $2.3 \%$ anti-oxidation, and $7.0 \%$ of them used other methods. The use of herbal remedies and traditional medicine is as important as the use of antiviral drugs. Furthermore, anti-viral and anti-inflammatory treatments are of great interest to the investigators. In this regard, the first anti-viral clinical trial with LPV/r has already reached the targeted sample size of 160 cases. The results of a systematic review using lopinavir therapy for SARS and MERS coronaviruses showed that lopinavir may be considered as a potential treatment option for the COVID19. ${ }^{19}$ Also, remdesivir, as an adenosine analog, was used for this virus treatment, and besides, the related clinical trials of this drug are ongoing. Moreover, remdesivir terminates the viral replications when incorporated into the nascent viral RNA chains. ${ }^{20}$

The national institute for the infectious diseases " $L$. Spallanzani”, IRCCS in Italy recommended to use LPV/r, the Darunavir or Tocilizumab alternatively depending on the symptoms and severity of the disease.

Besides the above-mentioned drugs, oseltamivir was suggested for the treatment of the COVID19 suspected children based on the results of an algorithm that was according to the standard diagnosis and treatment strategies for pediatric viral infections. ${ }^{21}$ However, some supportive therapies such as prompt availability of $\mathrm{O} 2$ should be done, in case of necessity. ${ }^{22}$ Another antiviral drug is the favipiravir, which targets the viral RNA polymerase; also, it is different from many influenza antivirals that target proteins on the surface of the virus like Roche's Tamiflu (oseltamivir phosphate). Since the SARS-CoV-2 RNA polymerase is similar enough to the influenza polymerase, this ability makes the favipiravir a potential drug for the COVID-19. ${ }^{23,24}$ Moreover, Favipiravir, also known as T-705, Avigan, or favilavir, is an antiviral drug being developed by the Toyama Chemical of Japan known with the activity against many RNA viruses. In the clinical trial, it was reported that this recommended drug leads to faster viral clearance compared to the LPV/r group, with a median of 4 days versus 11 days, respectively, as well as an improvement in chest imaging. However, a large sample size of the participants is needed to perform further clinical studies. ${ }^{25}$ Deng et $\mathrm{al}^{14}$ compared arbidol and (LPV/r) treatment with LPV/r alone for the patients with 
COVID-19 and then reported satisfactory clinical results with arbidol and LPV/r compared to LPV/r alone.

In a study, Wang et $\mathrm{al}^{26}$ evaluated the antiviral efficiency of five FAD-approved drugs including ribavirin, penciclovir, nitazoxanide, nafamostat, chloroquine as well as two wellknown broad-spectrum antiviral drugs remdesivir and favipiravir on a clinical isolate of COVID-2019. In this regard, favipiravir showed a significant effect in vitro and they suggested performing more in vivo studies to evaluate its antiviral property additionally. Also, other researchers conducted randomized clinical trials among the moderate COVID-19 patients who were previously untreated with antiviral. They reported that favipiravir could be considered as a preferred treatment due to the higher clinical recovery rate of day 7 , which reduced the incidence of fever and cough more effectively, except for the manageable antiviral-associated adverse effects. Moreover, this drug can better act compared to arbidol, which is the recommended drug in the Chinese diagnosis and treatment protocol for the novel Coronavirus Pneumonia. ${ }^{27}$ Also, there is pre-clinical evidence of effectiveness resulted from the use of chloroquine in the patients with COVID-19. Accordingly, chloroquine or hydroxychloroquine has shown an anti-SARS-CoV-2 activity under in vitro condition and have been used in the clinical trial research. ${ }^{26}$ The in vitro data suggest that chloroquine has an in vitro activity against many different viruses, which inhibits the SARS Cov-2 replication. Also, it is hypothesized that chloroquine prevents SARS-CoV-2 binding to target cells because it interferes with a virus-cell surface receptor, as the angiotensinconverting enzyme 2 (ACE2) receptor glycosylation. ${ }^{28}$ In addition, chloroquine, which is a broadly used antimalarial drug, has been proposed several times for the treatment of acute viral diseases in human being without success. ${ }^{29}$ However, poisoning that accrued with the toxic dose of chloroquine has been reported, which is also associated with cardiovascular disorders. ${ }^{30}$

Another compound named Thymoquinone has anti-sepsis and immunomodulatory activities at specific doses. Thymoquinone has been shown to downregulate inflammatory cytokines, reduce NO levels, and improve organ functions and survival of sepsis in an animal model. It has demonstrated that Thymoquinone, an emerging natural drug, is the main constituent of Nigella sativa and is anti-inflammatory, antioxidant, anti-tumor, and antimicrobial agent. ${ }^{31}$

In the anti-inflammatory or immunomodulation approach, such agents as corticosteroids, immunoglobins or antiinterleukin 6 are being used. Most of the deaths that accrue from infection are resulted by dysfunctions or failures of the lung or multiple organs, which are related to the host's immune dysfunction or the related disorders. Huang et al, ${ }^{32}$ using immune profiling analysis with single-cell resolution, demonstrated that the blood single-cell immune profile reveals the interferon-MAPK pathway is mediated by adaptive immune response for the COVID-19. Also, the interferonMAPK pathway is known as the major defense mechanism in the COVID-19 immune response. Moreover, they noted that the SARS-CoV-2 can cause a blood immune reaction after infecting the respiratory system. Explaining, it enters the blood through the circulatory system in the patients with COVID-19 and its damage would be related to multiple organs. ${ }^{33}$

The expression of the key transcription factors such as FOS, JUN, and JUNB through the downstream activation of MAPK causes the production of several effectors such as IFI27, IFITM1, and IFITM3, to fight the virus. Therefore, after the infection, a wide range of antiviral responses appears in the blood, while the MAPK signal is inhibited in the normal situation of the patient's recovery condition. Accordingly, their results showed that immune deficiency or imbalance adaptive immune response condition may worsen the COVID-19 patients' condition. $^{33-35}$

So, efforts to maintain a balanced and optimal immunity focusing on this pathway can help in the prevention, management, and treatment of this disease, as well as providing some important information for the drug development of COVID-19.

In agreement with the role of the interferon-MAPK pathway in the COVID-19 immune response, other researchers reported that IFN- $\alpha$, usually used to treat hepatitis, inhibits SARS-CoV reproduction under in vitro condition. Also, in the fifth edition of the guidelines, it has been recommended that the specific method for the administration of IFN- $\alpha$ is vapor inhalation combined with the therapy by antivirals including LPV/r, and ribavirin for the treatment of COVID-19. Also, other researchers suggested that the regulation of interferon production can be considered as a potential strategy for the COVID-19 treatment. $^{25,34}$ Furthermore, one of the other clinical trial methods, which have been applied against COVID-19 treatment, was the use of Cytokines directed antagonists such as adalimumab (TNF- $\alpha$ ) and CMAB806 (IL-6). In addition, upstream regulation of cytokines production could be regarded as a promising strategy for the treatment of COVID-19. For example, the approved drugs such as suramin and anaplastic lymphoma kinase inhibitors are worthy for clinical trials. $^{34}$ 
A valuable model for correlating the Pathogen Infection Recovery Probability (PIRP) in competition with Pro-inflammatory Anti-Pathogen Species (PIAPS) levels within a host unit was proposed by Shajing Sun. ${ }^{35}$ Correspondingly, the mentioned researcher has also reported that the maximum PIRP was exhibited when the PIAPS levels were either equal to or around the PIAPS equilibrium levels at the pathogen elimination or clearance onset. For performing a successful treatment, it is very important to improve the host's PIRP and more important to prevent the cytokine storm. So, such treatments are needed only for those hosts who have deficient or very weak anti-pathogen immune responses, which should be administered within the primary stage of infection in a controlled manner. The immune-modulatory therapy, with or without the combination of antiviral agents, may improve the outcome because the homeostasis of the immune system plays a key role in the development of COVID-19 pneumonia. ${ }^{36,37}$

In a multi-center study conducted on 416 cases of COVID-19 with the definite outcome from 14 hospitals in Hubei province, the clinical characteristics and treatment regimens up to Feb 17, 2020, were extracted. In this regard, the obtained data showed that $91 \%(380 / 416)$ of the patients were given the anti-viral therapy. While the rates of the corticosteroid therapy, $\gamma$-globulin, and invasive ventilation significantly increased as $84 \%, 67 \%, 24 \%$ for deaths and prolonged hospitalization for survivors who used corticosteroids, respectively. Also, corticosteroid therapy, $\gamma$-globulin, and invasive ventilation were the most frequently used in the death group. ${ }^{38}$

\section{Monoclonal Antibodies}

The major class of bio therapeutically methods and antiviral infection approach is passive immunotherapy using monoclonal antibodies whose therapeutic potential in the treatment of many diseases has been well recognized. Until now, there are no approved therapeutic drugs to treat coronavirus infection, so it is necessary to develop effective agents to have a successful therapy and also to prevent future deaths caused by this dangerous virus. On the other hand, several genetic data, clinical signs, and epidemiological features of COVID-19 resemble the SARS-CoV infection. Therefore, the previous advanced research works conducted on SARS-CoV treatment could help scientists to develop effective therapeutic strategies and novel drugs to treat this infection. ${ }^{39}$ Tian et $\mathrm{al}^{40}$ reported that CR3022, which is a SARS coronavirus- specific human monoclonal antibody, could be developed as a potential candidate for the treatment of SARS-CoV-2 infection, alone or in combination with other neutralizing antibodies. Moreover, CR3022 can effectively bind with the receptor-binding domain (RBD) of SARS-CoV-2, and its epitope has no overlap with the angiotensin-converting enzyme 2 (ACE2) binding site within 2019-nCoV RBD. Accordingly, in agreement with them, other researchers stated that other monoclonal antibodies neutralizing SARS-CoV such as m396 and CR3014, by targeting the ACE2 binding site of SARS-CoV, could be known as an alternative for the treatment of COVID-19. ${ }^{8,41}$ Since the alteration in the RBD of SARS-CoV and 2019-nCoV has a critical effect on the cross-reactivity of neutralizing antibodies, so the development of new monoclonal antibodies, which can exactly bind to $2019-\mathrm{nCoV}$ RBD, is needed.

\section{Alternative Medicine}

Herbal medicine, which was the only treatment available before the introduction of antibiotics, has been found to be effective in reducing infectious disorders. Nowadays, the use of natural products with anti-viral properties is increasingly growing among many populations worldwide, which provides a rich tool for the production of novel antiviral drugs. ${ }^{42}$

Several results obtained from research works and clinical trials showed that traditional Chinese medicine (TCM) plays a significant role in the prevention, control, and treatment of COVID-19. ${ }^{43}$ Interesting point is that onethird of the registered clinical trials on the COVID-19 patients used TCM therapy that has usually been used in combination with modern medicine. A meta-analysis study on TCM in the treatment of SARS suggested that this method could be effective in relieving the symptom of fever, which showed the requiring lesser dosage of corticosteroid and reduced pulmonary infiltration. Besides, the combination of TCM with modern medicine sounds hopeful for treating diseases caused by influenza and coronavirus. $^{44,45}$ In this regard, several doctors and researchers recommended that the chemical compounds required to cure COVID-19, could be found in natural products like tea. Dr. Li Wenliang had documented some case files on the use of tea by the patients who suffered from the COVID-19. Even the previously performed studies show that tea is an interesting natural substance possessing anti-viral activities in plants, animals, and human beings. ${ }^{46,47}$ 
Since tea is one of the most common and popular drinks worldwide, which is in harmony with everyone's nutritional habits, itself or its useful extracts could be used to improve this disease. ${ }^{48}$

In recent decades, the green tea catechins (GTCs) have been reported to provide various health benefits for numerous diseases. Correspondingly, they are polyphenolic compounds obtained from the leaves of Camellia sinensis, which have antiviral effects on diverse viruses. ${ }^{49}$ Besides, an investigation was performed on the anti-viral effect of the aflavins in tea, and the related results also showed that the aflavins manifest the inhibitory effects on infection and multiplication of virus, by bonding themselves to nucleic acids of the virus. ${ }^{50-52}$ By quantitative RT-PCR of the influenza virus-specific mRNA, the researchers reported that the GTCs can affect the transcription of viral genes in the infected cells. Also, they suggested that the GTCs have inhibitory activity on a viral attachment to the host cells. Accordingly, the primary target for GTCs is a membrane and physical integrity of the virus particles to the host membrane. Besides, the GTCs act on the acidification of intracellular endosome compartments, which are required for the fusion of viral and cellular membranes. ${ }^{53}$ Further studies also showed that the 3-galloyl group of GTC skeleton plays an important role in the activity of the virus, while the $5-\mathrm{OH}$ at the trihydroxy benzyl moiety at 2-position plays a minor role. $^{52}$ Runfeng et $\mathrm{al}^{54}$ reported that Lianhuaqingwen (LH), as TCM formula, significantly inhibits SARS-CoV -2 replication under in vitro condition, affects the virus morphology, and reduces the production of the proinflammatory cytokines at the mRNA levels. In a virtual screening study, some compounds were predicted to bind to the binding pocket of 3CLPro protease (3CLpro). In this regard, 3CLpro plays an important role in the replication of coronavirus, which can be considered as a potential drug target for the development of a drug against COVID-19. ${ }^{55}$

\section{Stem Cell Therapy and Plasma Containing Antibodies}

Mesenchymal stem cells (MSCs) have shown a powerful immunomodulatory function. Accordingly, researchers found that the MSC transplantation improves the clinical outcomes, as well as the changes of inflammatory and immune function levels. Moreover, they reported that the transplantation of ACE2 negative MSCs improves the outcome of the patients with COVID-19 pneumonia. In addition, the intravenous transplantation of MSCs was safe and effective, especially for the patients who are in critically severe conditions. ${ }^{56}$

Chen et $\mathrm{al}^{57}$ have conducted a clinical study of mesenchymal stem cell (MSC) therapy on the patients with epidemic Influenza $A$ (H7N9) infection who had acute respiratory distress syndrome (ARDS). In this regard, they suggested that this protocol may be effective in the treatment of COVID-19. Also, they found that the mortality was significantly lower in the experimental group compared to the control group. During the fiveyear follow-up period, MSC transplantation had no harmful effects on the bodies of some of the treated patients. Based on the similar complications (eg, ARDS and lung failure) of H7N9 and 2019-nCoV as well as the correspondence of multi-organ dysfunction, they suggested that MSC-based therapy could be considered as a possible alternative treatment to treat the COVID-19. A group of scientists suggested that the expanded umbilical cord MSCs (UC-MSCs) could be a therapeutic strategy in managing the critically ill COVID-19 patients to be compassionately used to reduce morbidity and mortality. Based on the preclinical and preliminary clinical data, they reported that the use of UCS-MCS through its antiinflammatory and immunomodulatory actions could heal tissues and enhance recovery. Additionally, they claimed that the UCS-MCS treatment could be regarded as an antimicrobial strategy. The MSCs affect the host immune response against pathogens such as increasing the activity of phagocytes, secretion of antimicrobial peptides and proteins, and the expression of molecules such as indoleamine 2, 3-dioxygenase (IDO) and interleukin (IL)-17. ${ }^{58}$

The convalescent plasma or immunoglobulins has been used to improve the survival rate of the patients with severe acute respiratory syndrome (SARS), the pandemic 2009 influenza A (H1N1), the avian influenza A (H5N1), several hemorrhagic fevers such as Ebola, and other viral infections. Accordingly, some clinical trials as well as a meta-analysis study have shown that the plasma donation of the patients recovered from the coronaviruses disease such as SARS-CoV and MERS-CoV, has had some favorable results in the treatment of other patients, which may reduce the mortality rate. So, in the other clinical trial way, the plasma containing antibodies that are developed during the convalescent phase from the infected patients are being used to treat COVID-19. The efficacy of these approaches is not clearly known yet, and the historical experience proposes that the convalescent sera may be more effective in preventing disease compared to 
treatment. $^{59-61}$ Also, a shorter hospital stay and a lower mortality rate have been observed in the patients who were treated compared to those who were not treated with convalescent plasma in the related clinical trials. However, there are still some challenges, specifically on COVID-19 treatment. The donated blood products would be screened for the infectious agents according to the current blood banking practices. Besides, individual sera would be correctly studied for specific antibody content and neutralizing activity to SARS-CoV-2. However, using old drugs for the treatment of COVID-19 currently is the only and available way, until finding an effective treatment. ${ }^{62}$

\section{Antiviral Drug Development Strategies}

In multiple structural and biochemical interaction studies, it was found that ACE2 is a key receptor for the spike glycoprotein of SARS-CoV-2. ${ }^{63}$

The $\mathrm{S}$ protein RBD domain of SARS-CoV-2 interacts with ACE2 and this interaction determines the host range and tropism. In this regard, the interaction between RBD of SARS-CoV-2/SARS-CoV and mammalian ACE2 was predicted by a sequence alignment study performed on the amino acids binding to RBD in ACE2. ${ }^{64}$ Also, the structural simulation results of SARS-CoV showed that N82 in ACE2 has a closer contact with F486 of SARS-CoV2 S protein compared to M82 of ACE2 (Figure 4).

Based on the above-mentioned studies, the focus of multiple drug development projects is on the ACE2-SARS-CoV-2 Spike interactions. $^{65,66}$ In a study, researchers showed that the human recombinant soluble ACE2 (hrsACE2) blocks the growth of SARS-CoV-2 in a dose-dependent manner, which also reduces the SARS-CoV-2 recovery from Vero cells by a factor of 1000-5000. Accordingly, they concluded that their study had some limitations, because the design of the study focused on the early stages of infection, which represents that hrsACE2 could block the early entry of SARS-CoV-2 infections in the host cells. So, to illuminate the effect of hrsACE2 at the later stages of infection, performing further in vitro and in vivo studies are needed. Also, lung organoids should be carefully studied, because the lung is the major target organ for COVID-19. ${ }^{67}$

The SARS-CoV-2 main protease (Mpro) is known as another target that has been recently suggested for the development of the novel drug candidates for COVID-19. In a deep docking of 1.3 billion compounds obtained from ZINC15 library, 1, 000 potential inhibitors of SARS-CoV-2 Mpro have been selected for further characterization and development. $^{68}$
A

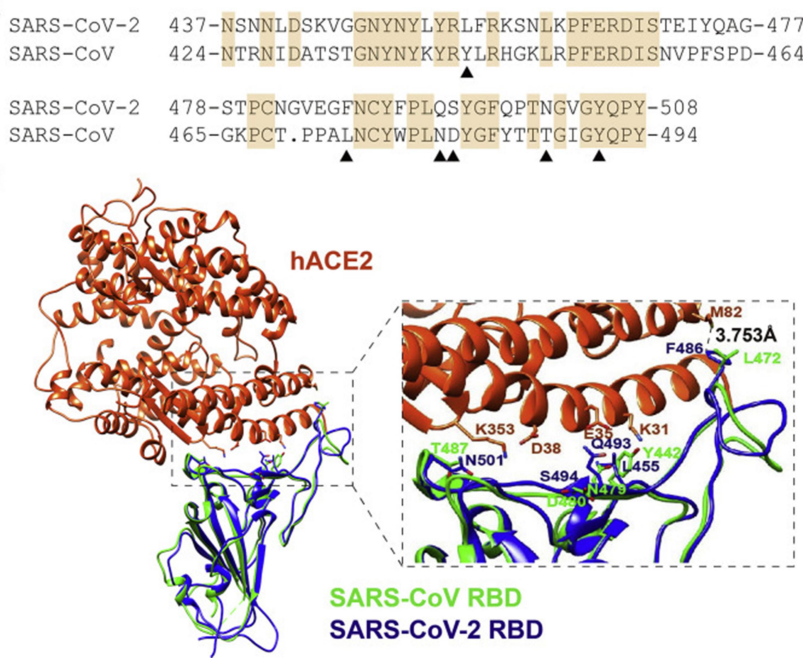

Figure 4 The RBM region of S proteins from SARS-CoV-2 and SARS-CoV alignment. Notes: (A) The six key amino acids in the S protein interacting with human ACE2 shown by black triangles. (B) Structural Alignment of the ACE2 recognition of RBD from SARS-CoV-2 and SARS-CoV. Human ACE2 (hACE2), SARS-CoV-2 RBD, and SARS-CoV RBD are in orange-red, blue, and green, respectively. Reprinted from Biochemical and Biophysical Research Communications, Vol 526/ Edition I, Luan J, Lu Y, Jin X, Zhang L, Spike protein recognition of mammalian ACE2 predicts the host range and an optimized ACE2 for SARS-CoV-2 infection, Pages No.165-169, Copyright (2020), with permission from Elsevier. ${ }^{64}$

Some other agents such as the statins, angiotensin receptor blockers (ARBs), and angiotensin-converting enzyme inhibitors (ACEIs), have been suggested to treat SARS-CoV-2 infection. Based on the epidemiological data, over two-thirds of the patients died from COVID-19 had diabetes or cardiovascular diseases. Accordingly, they were treated by angiotensin-receptor blockers (ARBs) as a first-line therapy. Moreover, there are several known indications that ARBs can more significantly increase the ACE2 expression in the kidney and the heart (2- to 5-fold); whereas, regarding lungs, there is no direct evidence yet. A study showed that ACE2 could be expressed in lower lungs on type I and II alveolar epithelial cells of normal human lungs. In addition, ACE2 has a high expression in the mouth and tongue that facilitates viral entry in the host. So, those ARBs can increase ACE most probably even in alveolar cells. It may be wondering that how the use of these drugs could predispose the patients to more severe illness and the increased SARS-CoV-2 infection. ${ }^{69}$ On the other hand, preclinical studies conducted on different animal models of severe lung injury have demonstrated that ACE2 is substantially downregulated. Also, the use of losartan reduces severe acute lung injury in mice injected by the spike glycoprotein of SARS-CoV. Due to these contradictory findings, one cannot explain clearly the protective effect of ACE2 upregulation by ARBs during COVID-19 infection. 


\section{Nano-Based Drug Delivery Systems}

Nanotechnology, as an advanced therapeutic option, was shown in recent studies to play a great potential role against COVID-19 such as vaccine development and using engineered nanocarriers in drug delivery systems. Therefore, in this regard, it is important to look and select a suitable nanocarrier delivery system to find a safe and effective treatment. ${ }^{70}$ All of Nano medicine strategies for COVID-19 therapeutics and vaccine development are designed on the key of target identification, in order to stop or block the pathogenesis of the viral infection. Accordingly, the major identified targets are viral protease, host cell produced protease, viral RNA polymerase, and the interaction site of viral S protein with host receptor ACE2. ${ }^{71-74}$ Moreover, another strategy proposed for COVID-19 treatment is targeting the SARS-CoV-2 surface $S$ protein using neutralizing antibody (nAbs) ${ }^{75}$ Besides, targeting the SARS-CoV-2 viral RNA genome by the use of RNA interference (RNAi) or antisense oligonucleotides is known as another interesting approach to have a potential therapy of this disease. ${ }^{76,77}$ In Nano-based drug delivery systems, several evidences indicated that multiple nanomaterials such as graphene, nanodiamonds, carbon nanotubes, and polystyrene particles, have an intrinsic capacity to act as anti COVID-19 agents, especially via activating the immune system, which mostly depends on their functionalization. ${ }^{78}$ Several other studies have also shown that extracellular vehicles (EVs), which are a family of natural carriers in the human body, play critical roles in cellto-cell communications. Moreover, they can be used as unique drug carriers to deliver protease inhibitors to treat COVID-19 with fewer systemic side effects. ${ }^{79}$ Although several researchers in their studies proposed that nanotechnology can provide biosensors, vaccines, and antiviral materials, all of these studies should be developed in in vitro and in vivo expanded investigations as well as a proper formulation to be confirmed for clinical trials usage.

\section{Discussion About Suggested Strategy}

Over $30 \%$ of the treated patients do not achieve remission because of the body's inflammatory response, which is known as one of the causes of depression. On the other hand, the nervous and immune systems have interactions with each other. In the patients suffering from depression, changes in the plasma concentrations of cytokines as well as the number and level of activation of immune cells have been found (Figure 5). Moreover, neurotransmitters are

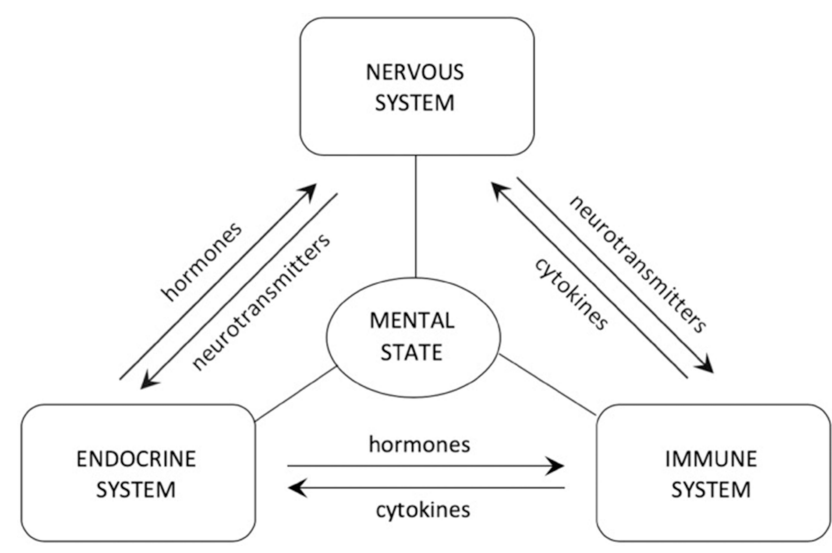

Figure 5 The interaction among nervous, endocrine, and immune systems, modulated by the secreted hormones, neurotransmitters, and cytokines.

Notes: Reproduced from Szałach $Ł P$, Lisowska KA, Cubała WJ. The influence of antidepressants on the immune system. Arch Immunol Ther Exp (Warsz). 2019;67(3):143-151. ${ }^{86}$

signal substances and mere mediators of signal states among the cells in the nervous system. Several pieces of evidence elucidated the modulatory role of neurotransmitters in immune function, the regulation of migration of leukocytes, and even tumor cells. Serotonergic and dopaminergic pathways are attractive targets to treat human immune, infectious, and cancerous diseases. ${ }^{80,81}$ In several successful investigations, agonists or antagonists of dopamine or serotonin receptors have been used for the activation of autophagy or the induction of apoptosis. ${ }^{82-84}$ In these pathways, serotonin has a stimulatory effect on the cytotoxic $\mathrm{T}$ cells, plays a role in the activation of NK cell, and also increases the proliferative activity of B cells. Dopamine is a catecholamine neurotransmitter, which is synthesized in the brain and widely known as an important factor in the regulation of the homeostasis of immune, renal, hormonal, and central nervous systems. In the interim guidance document of WHO, when the novel coronavirus was suspected, it was mentioned that utilizing vasopressors (norepinephrine, epinephrine, and dopamine most safely received from a central venous catheter) helps clinicians in managing the patients with acute respiratory failure and septic shock, as a consequence of severe infection. ${ }^{57,85,86}$

Based on the described role of host immune responses, in overcoming the infection disease, it has been recommended that serotonergic and dopaminergic pathways are attractive targets to treat the human infectious disease. For example, fluoxetine, by its action on the serotonergic pathway in the central nervous system, regulates the neuroendocrine signals and also modulates the immune response against infection. In addition, the administration of fluoxetine would reduce the lymphocyte activity in the high basal immune function 
as well as improving the immune function when it is deficient by direct and indirect mechanisms. The indirect mechanisms would be either 5-HT-dependent (a group of $\mathrm{G}$ protein-coupled receptor named as 5-hydroxytryptamine receptors, 5-HT receptors, or serotonin receptors) or independent (Figure 6). It was shown that the concentration of this drug dose usage can affect the mechanism of its action. So, the results of the studies highlighted the importance of the novel pharmacological action of fluoxetine, as a novel immune-modulatory drug. Besides, fluoxetine could directly act on the $\mathrm{T}$ lymphocytes and dually modulate their proliferation, depending on the cellular activation. Fluoxetine, as one of the selective 5-HT reuptake inhibitors (SSRIs), has been demonstrated to be very efficient and safe with some low side effects. Moreover, fluoxetine and all other SSRIs are 5-HT2B agonists that are important for their therapeutic effects. In addition, fluoxetine is usually chosen for the treatment of depression symptoms, obsessive-compulsive disorder, panic attacks, and bulimia nervosa. The SSRIs are frequently used in antidepressant therapy as well as immune function modifications. In this regard, fluoxetine showed a novel pharmacological action as an immune modulator, which helps in the treatment of several pathogenesis immune deficiencies and/or in the presence of any deregulation. ${ }^{87}$

There are several reports on the advantage of hostdirected therapies (HDTs) for infections like MERS-CoV. Correspondingly, these benefits are immune mechanism protection, modulation of the destructive immune-mediated inflammatory responses, prevention of cytokine storm, and protection of tissues from inflammatory damage. ${ }^{88}$ Thus, the supportive and successful care of COVID19 is the main goal of treatment. In this way, prevention of these complications, particularly organ failure, ARDS, and secondary bacterial infections should also be considered. In addition, the modulation of immune signaling may significantly affect the outcome of this disease. Petersen et $\mathrm{al}^{89}$ reported that there were commonly used drugs with the good safety profiles that can be used in HDTs, and supplement host innate and adaptive immune mechanisms to MERS-CoV. Nevertheless, they suggested carefully performing the controlled trials to determine this relationship.

In a meta-analysis study, researchers analyzed data of 7 studies with a total of 662 Hepatitis C patients and reported that prophylactic SSRIs reduced the risk of depression. The incidence of IFN-induced major depression and depression severity were defined as primary and sustained virologic response is secondary outcomes. They found that SSRIs prevent interferon-a-induced depression in patients with Hepatitis C. ${ }^{90}$

An interesting report states that for some COVID-19 patients, reduction in tryptophan (TRP), the precursor of serotonin, levels may either expose an underlying vulnerability to depression or trigger a de novo episode of depression. This commentary discussed the pathway involved and recommended in-hospital augmentation with foods or supplements that increase TRP levels for COVID-19 patients treated with INFs and recommended that SSRIs may also could be tried. ${ }^{91}$ Since fluoxetine is a specific and potent inhibitor of the presynaptic reuptake of serotonin, works by increasing the amount of serotonin could be useful in the treatment of respected COVID-19 major depression. ${ }^{92}$

Molecular screening of small molecule libraries identified that fluoxetine was a potent inhibitor of coxsackievirus replication, which reduced the synthesis of viral RNA and protein. ${ }^{93}$ Immunomodulatory properties of fluoxetine have

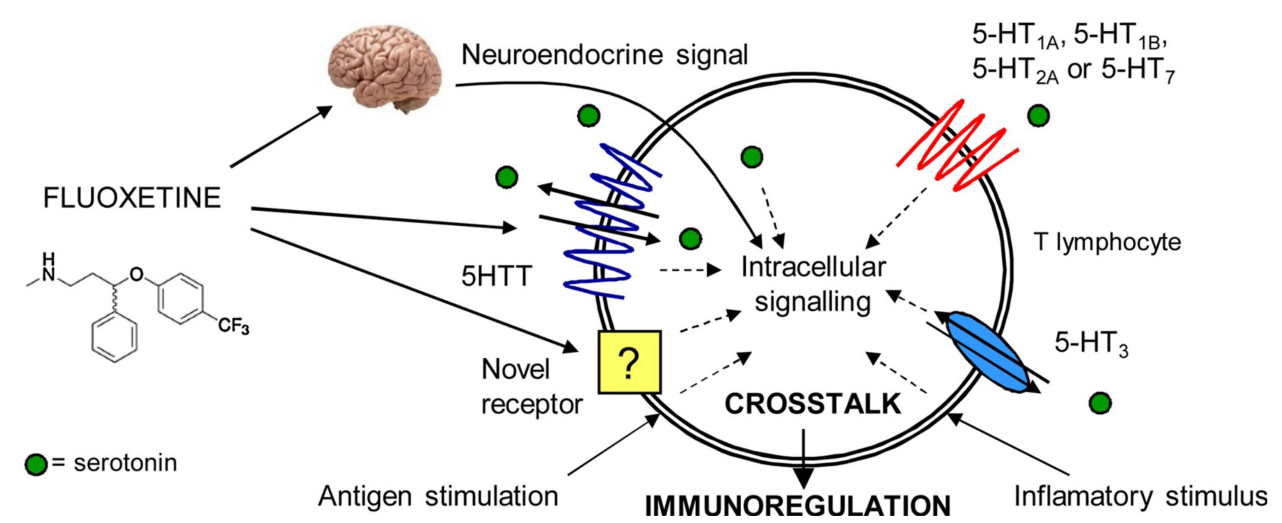

Figure 6 The crosstalk effect of fluoxetine in intracellular signaling and its immune-regulatory role.

Notes: Reprinted from Pharmacological Research, Vol 109, Di Rosso ME, Palumbo ML, Genaro AM, Immunomodulatory effects of fluoxetine: a new potential pharmacological action for a classic antidepressant drug?, Pages No.10I-107, Copyright (2016), with permission from Elsevier. ${ }^{87}$ 
also been reported in some animal and human models. The host-targeted small molecules modulate excessive inflammation and reduce lung tissue destruction. Also, researchers reported that fluoxetine, as a host-targeted small molecule, restricts the growth of intracellular Mycobacterium tuberculosis as well as inducing autophagy in the infected macrophages. ${ }^{94,95}$ Ulferts et al ${ }^{96}$ demonstrated that SSIR fluoxetine inhibited the replication of human Enteroviruses $B$ and $D$ by targeting viral protein.

Due to the ability of fluoxetine to enter the cell, even its currency through the new receptors, it could be used to compete with the virus to enter the cell and prevent the binding of SARS-CoV-2 to the target cells. Indeed, coadministration of fluoxetine in the treatment of COVID19 could be considered due to the possibility of its interaction with ACE2 receptors, immune-modulatory function, and a proper immune response at the right time. Moreover, it can compete with the virus to enter the cell and help in the regulation of the body's immune system against the virus. The Centers for Disease Control and Prevention (CDC) reported that the outbreak of coronavirus disease 2019 (COVID-19) may be stressful for people, which cause strong emotions in adults and children. Also, a document has been developed by the WHO department of mental health with a series of messages to support the mental and psychosocial well-being in different target groups during the outbreak of COVID19. ${ }^{97}$ As the influence of antidepressant drugs on the immune system was earlier demonstrated, the fluoxetine combination treatment may not only reduce the stress of the virus outbreak, but also decrease viral load to prevent and cure the infection. It seems that the use of this drug would be useful and effective, especially for the people near the patients because of care or therapy of them such as doctors, nurses or other treatment staff, and also their families. However, the accurate laboratory and clinical research are needed to prove these hypotheses based on the previous strong studies.

\section{Conclusion}

The outbreak of the new coronavirus is a major concern for human life. The high mortality rate and significant deaths caused by this virus are threats for international and global unity and cooperation. To deliver access to drugs and to find a cure as soon as possible, the entire world must work together. As a general summary of significant studies, about $50 \%$ of the clinical trials and treatment strategies are dedicated to use the antiviral and anti-inflammatory drugs. Notably, the use of traditional medicine is about $35 \%$, which indicates a tendency to use herbal medicines. Perhaps, it is because of the higher confidence in the low risk of side effects or their long-term consequences.

The coronavirus pandemic makes the progression of clinical studies slow with a long period. In this critical situation, the most accessible facilities and the most likely ways with a safer practical background must be paid attention. It is better to use the costly and late-yielding methods and external factors that interfere with the body's natural systems, which have not long-term effects evidence yet. Given the challenges ahead for some of these methods including cell therapy, recombinant factors, or even plasma transmission, it is best to deal with and overcome this emerging virus through biomimetic and the mechanisms of virus and host interaction.

So, the approved antiviral drugs and immunemodulatory agents combined with natural compounds are considered in the first line of accessible and beneficial treatments. Accordingly, this treatment method is the best because of large existence studies, long time follow-up, and proximity to the natural system, and the normal physiological routine of pathogen and host interactions.

\section{Acknowledgments}

I would like to thank deeply all the people of the world who patiently follow the safety tips during the pandemic of this virus. I thank those people who consider themselves obliged to prevent the spread of this disease and who are honest and kind in helping their fellow human beings. Moreover, I would especially like to thank the medical staffs who are enduring all the hardships to save the lives of the patients. Through this writing, I would like to appreciate and regard those who lost their lives to save the lives of the patients.

\section{Disclosure}

The author reports no conflicts of interest in this work.

\section{References}

1. Zhu N, Zhang D, Wang W, et al. China novel coronavirus investigating and research team. A novel coronavirus from patients with pneumonia in China, 2019. N Engl J Med. 2020;382(8):727-733. doi:10.1056/ NEJMoa2001017

2. Gralinski LE, Menachery VD. Return of the Coronavirus: 2019-nCoV. Viruses. 2020;12(2):135. doi:10.3390/v12020135

3. Shimizu K. 2019-nCoV, fake news, and racism. Lancet. 2020;395 (10225):685-686. doi:10.1016/S0140-6736(20)30357-3

4. Li Q, Guan X, Wu P, et al. Early transmission dynamics in Wuhan, China, of novel coronavirus-infected pneumonia. $N$ Engl J Med. 2020;382(13):1199-1207. doi:10.1056/NEJMoa2001316 
5. Rosa SGV, Santos WC. Clinical trials on drug repositioning for COVID-19 treatment. Revista Panamericana de Salud Pública. 2020;44:e40. doi:10.26633/RPSP.2020.40

6. Zhu R-F, Gao R-L, Robert S-H, Gao J-P, Yang S-G, Zhu C. Systematic review of the registered clinical trials of coronavirus diseases 2019 (COVID-19). medRxiv. 2020.

7. Cortegiani A, Ingoglia G, Ippolito M, Giarratano A, Einav S. A systematic review on the efficacy and safety of chloroquine for the treatment of COVID-19. J Crit Care. 2020.

8. Li X, Geng M, Peng Y, Meng L, Lu S. Molecular immune pathogenesis and diagnosis of COVID-19. J Pharm Anal. 2020;10(2):102-108. doi:10.1016/j.jpha.2020.03.001

9. Masters PS. The molecular biology of coronaviruses. Adv Virus Res. 2006;66:193-292.

10. Arafah A, Ali S, Yatoo AM, Ali MN, Rehman MU. S1 subunit and host proteases as potential therapeutic avenues for the treatment of COVID-19. Arch Med Res. 2020. doi:10.1016/j.arcmed.2020.05.013

11. Manhas S, Anjali A, Mansoor S, et al. Covid-19 pandemic and current medical interventions. Arch Med Res. 2020;51(6):473-481. doi:10.1016/j.arcmed.2020.05.007

12. Bolcato G, Bissaro M, Pavan M, Sturlese M, Moro S Targeting the coronavirus SARS-CoV-2: computational insights into the mechanism of action of the protease inhibitors Lopinavir, Ritonavir, and Nelfinavir. 2020.

13. Lim J, Jeon S, Shin H-Y, et al. Case of the index patient who caused tertiary transmission of COVID-19 infection in Korea: the application of lopinavir/ritonavir for the treatment of COVID-19 infected pneumonia monitored by quantitative RT-PCR. J Korean Med Sci. 2020;35(6).

14. Deng L, Li C, Zeng Q, et al. Arbidol combined with LPV/r versus LPV/r alone against Corona Virus Disease 2019: a retrospective cohort study. $J$ Infect. 2020;81(1):e1-e5. doi:10.1016/j. jinf.2020.03.002

15. Belhadi D, Peiffer-Smadja N, Yazdanpanah Y, Mentré F, Laouénan C. A brief review of antiviral drugs evaluated in registered clinical trials for COVID-19. medRxiv. 2020.

16. Lim J, Jeon S, Shin HY, et al. Case of the index patient who caused tertiary transmission of coronavirus disease 2019 in Korea: the application of lopinavir/ritonavir for the treatment of COVID-19 pneumonia monitored by quantitative RT-PCR. J Korean Med Sci. 2020;35(6).

17. Lim J, Jeon S, Shin HY, et al. The author's response: case of the index patient who caused tertiary transmission of coronavirus disease 2019 in Korea: the application of lopinavir/ritonavir for the treatment of COVID-19 pneumonia monitored by quantitative RT-PCR. $J$ Korean Med Sci. 2020;35(7).

18. Zhang T, He Y, Xu W, Ma A, Yang Y, Xu K-F. Clinical trials for the treatment of Coronavirus disease 2019 (COVID-19): a rapid response to urgent need. Sci China Life Sci. 2020;1-3. doi:10.1007/s11427020-1662-X

19. Yao TT, Qian JD, Zhu WY, Wang Y, Wang GQ. A systematic review of lopinavir therapy for SARS coronavirus and MERS coronavirus a possible reference for coronavirus disease-19 treatment option. J Med Virol. 2020;92(6):556-563. doi:10.1002/jmv.25729

20. Agostini ML, Andres EL, Sims AC, et al. Coronavirus susceptibility to the antiviral remdesivir (GS-5734) is mediated by the viral polymerase and the proofreading exoribonuclease. MBio. 2018;9(2): e00221-18. doi:10.1128/mBio.00221-18

21. Karimi A, Rafiei Tabatabaei S, Rajabnejad M, et al. An algorithmic approach to diagnosis and treatment of coronavirus disease 2019 (COVID-19) in children: Iranian expert's consensus statement. Arch Pediatr Infect Dis. 2020;8(2):e102400.

22. Nicastri E, Petrosillo N, Bartoli TA, et al. National Institute for the Infectious Diseases "L. Spallanzani", IRCCS. Recommendations for COVID-19 clinical management. Infect Dis Rep. 2020;12(1). doi:10.4081/idr.2020.8543
23. Goldhill DH, Te Velthuis AJ, Fletcher RA, et al. The mechanism of resistance to favipiravir in influenza. Proc Natl Acad Sci. 2018;115 (45):11613-11618. doi:10.1073/pnas.1811345115

24. Cai Q, Yang M, Liu D, et al. Experimental treatment with favipiravir for COVID-19: an open-label control study. Engineering. 2020. doi:10.1016/j.eng.2020.03.007

25. Dong L, Hu S, Gao J. Discovering drugs to treat coronavirus disease 2019 (COVID-19). Drug Discov Ther. 2020;14(1):58-60. doi: $10.5582 /$ ddt.2020.01012

26. Wang M, Cao R, Zhang L, et al. Remdesivir and chloroquine effectively inhibit the recently emerged novel coronavirus $(2019-\mathrm{nCoV})$ in vitro. Cell Res. 2020;30(3):269-271. doi:10.1038/s41422-020-0282-0

27. Chen C, Huang J, Cheng Z, et al. Favipiravir versus Arbidol for COVID-19: a randomized clinical trial. medRxiv. 2020.

28. Devaux CA, Rolain J-M, Colson P, Raoult D. New insights on the antiviral effects of chloroquine against coronavirus: what to expect for COVID-19? Int $J$ Antimicrob Agents. 2020;55(5):105938. doi:10.1016/j.ijantimicag.2020.105938

29. Touret F, de Lamballerie X. Of chloroquine and COVID-19. Antiviral Res. 2020;104762.

30. Frisk-Holmberg M, Bergqvist Y, Englund U. Chloroquine intoxication. Br J Clin Pharmacol. 1983;15(4):502. doi:10.1111/ j.1365-2125.1983.tb01540.x

31. Ahmad A, Rehman MU, Ahmad P, Alkharfy KM. Covid-19 and thymoquinone: connecting the dots. Phytother Res. 2020. doi:10.1002/ptr.6793

32. Huang L, Shi Y, Gong B, et al. Blood single cell immune profiling reveals the interferon-MAPK pathway mediated adaptive immune response for COVID-19. medRxiv. 2020.

33. Yang X, Yu Y, Xu J, et al. Clinical course and outcomes of critically ill patients with SARS-CoV-2 pneumonia in Wuhan, China: a single-centered, retrospective, observational study. Lancet Respir Med. 2020;8(5):475-481. doi:10.1016/S2213-2600(20)30079-5

34. Deng X, Yu X, Pei J. Regulation of interferon production as a potential strategy for COVID-19 treatment. arXiv Preprint arXiv:200300751. 2020.

35. Sun -S-S. Pathogen infection recovery probability (PIRP) versus proinflammatory anti-pathogen species (PIAPS) levels: modelling and therapeutic strategies. arXiv Preprint arXiv:200305507. 2020.

36. Liu Q, Zhou Y-H, Yang ZQ. The cytokine storm of severe influenza and development of immunomodulatory therapy. Cell Mol Immunol. 2016;13(1):3-10. doi:10.1038/cmi.2015.74

37. Zheng HY, Zhang M, Yang CX, et al. Elevated exhaustion levels and reduced functional diversity of $\mathrm{T}$ cells in peripheral blood may predict severe progression in COVID-19 patients. Cell Mol Immunol. 2020.

38. Shang J, Du R, Lu Q, et al. The treatment and outcomes of patients with COVID-19 in Hubei, China: a multi-centered, retrospective, observational study. 2020.

39. Shanmugaraj B, Siriwattananon K, Wangkanont K, Phoolcharoen W. Perspectives on monoclonal antibody therapy as potential therapeutic intervention for Coronavirus disease-19 (COVID-19). Asian Pac J Allergy Immunol. 2020;38(1):10-18.

40. Tian X, Li C, Huang A, et al. Potent binding of 2019 novel coronavirus spike protein by a SARS coronavirus-specific human monoclonal antibody. Emerg Microbes Infect. 2020;9(1):382-385. doi:10.1080/22221751.2020.1729069

41. Zhang L, Liu Y. Potential interventions for novel coronavirus in China: a systematic review. J Med Virol. 2020.

42. Ahmad A, Rehman M, Alkharfy K. An alternative approach to minimize the risk of coronavirus (Covid-19) and similar infections. Eur Rev Med Pharmacol Sci. 2020;24(7):4030-4034.

43. Ren J, Zhang A-H, Wang X-J. Traditional Chinese medicine for COVID-19 treatment. Pharmacol Res. 2020;155:104743. doi:10.1016/j.phrs.2020.104743

44. Chen Y, Guo JJ, Healy DP, Zhan S. Effect of integrated traditional Chinese medicine and western medicine on the treatment of severe acute respiratory syndrome: a meta-analysis. Pharm Pract. 2007;5(1):1-9. 
45. Onishi S, Mori T, Kanbara H, et al. Green tea catechins adsorbed on the murine pharyngeal mucosa reduce influenza A virus infection. J Funct Foods. 2020;68:103894. doi:10.1016/j.jff.2020.103894

46. Chen C-N, Lin CP, Huang -K-K, et al. Inhibition of SARS-CoV 3C-like protease activity by theaflavin-3, 3'-digallate (TF3). Evid Based Complement Alternat Med. 2005;2(2):209-215. doi:10.1093/ecam/neh081

47. Matsumoto M, Mukai T, Furukawa S, Ohori H. Inhibitory effects of epigallocatechin gallate on the propagation of bovine coronavirus in Madin-Darby bovine kidney cells. Anim Sci J. 2005;76(5):507-512. doi:10.1111/j.1740-0929.2005.00297.x

48. Lee YH, Jang YH, Kim Y-S, Kim J, Seong BL. Evaluation of green tea extract as a safe personal hygiene against viral infections. $J$ Biol Eng. 2018;12(1):1. doi:10.1186/s13036-017-0092-1

49. Mahmood MS, Mártinez JL, Aslam A, et al. Antiviral effects of green tea (Camellia sinensis) against pathogenic viruses in human and animals (a mini-review). Afr J Tradit Complement Altern Med. 2016;13 (2):176-184. doi:10.4314/ajtcam.v13i2.21

50. Okada F. Antiviral effects of tea catechins and black tea theaflavins on plant viruses. JARQ. 1978;12:27-32.

51. Xu J, Xu Z, Zheng W. A review of the antiviral role of green tea catechins. Molecules. 2017;22(8):1337.

52. Song J-M, Lee K-H, Seong B-L. Antiviral effect of catechins in green tea on influenza virus. Antiviral Res. 2005;68(2):66-74. doi:10.1016/ j.antiviral.2005.06.010

53. Imanishi N, Tuji Y, Katada Y, et al. Additional inhibitory effect of tea extract on the growth of influenza A and B viruses in MDCK cells. Microbiol Immunol. 2002;46(7):491-494. doi:10.1111/j.13480421.2002.tb02724.x

54. Runfeng L, Yunlong $\mathrm{H}$, Jicheng $\mathrm{H}$, et al. Lianhuaqingwen exerts anti-viral and anti-inflammatory activity against novel coronavirus (SARS-CoV-2). Pharmacol Res. 2020;156:104761. doi:10.1016/j. phrs.2020.104761

55. Sun N, Wong WL, Guo J. Prediction of potential 3CLpro-targeting anti-SARS-CoV-2 compounds from Chinese medicine. 2020.

56. Leng Z, Zhu R, Hou W, et al. Transplantation of ACE2-mesenchymal stem cells improves the outcome of patients with COVID-19 pneumonia. Aging Dis. 2020;11(2):216-228. doi:10.14336/ AD.2020.0228

57. Chen J, Hu C, Chen L, et al. Clinical study of mesenchymal stem cell treatment for acute respiratory distress syndrome induced by epidemic influenza A (H7N9) infection: a hint for COVID-19 treatment. Engineering. 2020. doi:10.1016/j.eng.2020.02.006

58. Orleans L, Is Vice H, Manchikanti L. Expanded umbilical cord mesenchymal stem cells (UC-MSCs) as a therapeutic strategy in managing critically Ill COVID-19 patients: the case for compassionate use. Pain Physician. 2020;23:E71-E83.

59. Mair-Jenkins J, Saavedra-Campos M, Baillie JK, et al. The effectiveness of convalescent plasma and hyperimmune immunoglobulin for the treatment of severe acute respiratory infections of viral etiology: a systematic review and exploratory meta-analysis. $J$ Infect Dis. 2015;211(1):80-90. doi:10.1093/infdis/jiu396

60. Casadevall A, Pirofski LA. The convalescent sera option for containing COVID-19. J Clin Invest. 2020;130(4):1545-1548. doi:10.1172/ JCI138003

61. Shen C, Wang Z, Zhao F, et al. Treatment of 5 critically Ill patients with COVID-19 with convalescent plasma. JAMA. 2020.

62. Chen L, Xiong J, Bao L, Shi Y. Convalescent plasma as a potential therapy for COVID-19. Lancet Infect Dis. 2020;20(4):398-400. doi:10.1016/S1473-3099(20)30141-9

63. Kuhn JH, Radoshitzky SR, Li W, Wong SK, Choe H, Farzan M. The SARS coronavirus receptor ACE 2 A potential target for antiviral therapy. New Concepts Antivir Ther. 2006;397-418.

64. Luan J, Lu Y, Jin X, Zhang L. Spike protein recognition of mammalian ACE2 predicts the host range and an optimized ACE2 for SARS-CoV-2 infection. Biochem Biophys Res Commun. 2020;526 (1):165-169. doi:10.1016/j.bbrc.2020.03.047
65. Zhou P, Yang X-L, Wang X-G, et al. A pneumonia outbreak associated with a new coronavirus of probable bat origin. Nature. 2020;579(7798):270-273.

66. Wrapp D, Wang N, Corbett KS, et al. Cryo-EM structure of the 2019-nCoV spike in the prefusion conformation. Science. 2020;367 (6483):1260-1263. doi:10.1126/science.abb2507

67. Monteil V, Kwon H, Prado P, et al. Inhibition of SARS-CoV-2 infections in engineered human tissues using clinical-grade soluble human ACE2. Cell. 2020;181(4):905-913.e7. doi:10.1016/j.cell.2020.04.004

68. Ton A-T, Gentile F, Hsing M, Ban F, Cherkasov A. Rapid identification of potential inhibitors of SARS-CoV-2 main protease by deep docking of 1.3 billion compounds. Mol Inform. 2020;39(8):2000028. doi:10.1002/minf.202000028

69. Fang L, Karakiulakis G, Roth M. Are patients with hypertension and diabetes mellitus at increased risk for COVID-19 infection? Lancet Respir Med. 2020;8(4):e21. doi:10.1016/S2213-2600(20)30116-8

70. Chauhan G, Madou MJ, Kalra S, Chopra V, Ghosh D, MartinezChapa SO. Nanotechnology for COVID-19: therapeutics and vaccine research. ACS Nano. 2020.

71. Du L, He Y, Zhou Y, Liu S, Zheng B-J JS. The spike protein of SARS-CoV - a target for vaccine and therapeutic development. Nat Rev Microbiol. 2009;7(3):226-236.

72. Lung J, Lin YS, Yang YH, et al. The potential chemical structure of anti-SARS-CoV-2 RNA-dependent RNA polymerase. J Med Virol. 2020;92(6):693-697. doi:10.1002/jmv.25761

73. Hoffmann M, Kleine-Weber H, Schroeder S, et al. SARS-CoV-2 cell entry depends on ACE2 and TMPRSS2 and is blocked by a clinically proven protease inhibitor. Cell. 2020;181(2):271-280.e8. doi:10.1016/j.cell.2020.02.052

74. Báez-Santos YM, John SES, Mesecar AD. The SARS-coronavirus papain-like protease: structure, function and inhibition by designed antiviral compounds. Antiviral Res. 2015;115:21-38. doi:10.1016/j. antiviral.2014.12.015

75. Shin YW, Chang K-H, Hong G-W, et al. Selection of vaccinia virus-neutralizing antibody from a phage-display human-antibody library. J Microbiol Biotechnol. 2019;29(4):651-657.

76. Ghosh S, Firdous SM, Nath A. siRNA could be a potential therapy for COVID-19. EXCLI J. 2020;19:528.

77. Chowdhury UF, Shohan MUS, Hoque KI, Beg MA, Siam MKS, Moni MA. A computational approach to design potential siRNA molecules as a prospective tool for silencing nucleocapsid phosphoprotein and surface glycoprotein gene of SARS-CoV-2. bioRxiv. 2020.

78. Weiss C, Carriere M, Fusco L, et al. Toward nanotechnology-enabled approaches against the COVID-19 pandemic. ACS Nano. 2020;14 (6):6383-6406. doi:10.1021/acsnano.0c03697

79. Kumar S, Zhi K, Mukherji A, Gerth K. Repurposing antiviral protease inhibitors using extracellular vesicles for potential therapy of COVID-19. Viruses. 2020;12(5):486. doi:10.3390/v12050486

80. Shaikhpoor M, Ahangari G, Sadeghizadeh M, Khosravi A, Derakhshani Deilami G. Significant changes in D2-like dopamine gene receptors expression associated with non-small-cell lung cancer: could it be of potential use in the design of future therapeutic strategies? Curr Cancer Ther Rev. 2012;8(4):304-310.

81. Entschladen F, Lang K, Drell TL, Joseph J, Zaenker KS. Neurotransmitters are regulators for the migration of tumor cells and leukocytes. Cancer Immunol Immunother. 2002;51(9):467-482. doi:10.1007/s00262-002-0300-8

82. Sheikhpour M, Sadeghizadeh M, Yazdian F, et al. Co-administration of curcumin and bromocriptine nano-liposomes for induction of apoptosis in lung cancer cells. Iran Biomed J. 2020;24(1):24. doi:10.29252/ibj.24.1.24

83. Sheikhpour M, Ahangari G, Sadeghizadeh M, Deezagi A. A novel report of apoptosis in human lung carcinoma cells using selective agonist of D2-like dopamine receptors: a new approach for the treatment of human non-small cell lung cancer. Int J Immunopathol Pharmacol. 2013;26(2):393-402. doi:10.1177/039463201302600212 
84. Rein T. Is autophagy involved in the diverse effects of antidepressants? Cells. 2019;8(1):44. doi:10.3390/cells8010044

85. Read MC. Clinical management of severe acute respiratory infections when novel coronavirus is suspected: what to do and what not to do (WHO, Feb 21 2013). World Health. 2013;4:22.

86. Szałach ŁP, Lisowska KA, Cubała WJ. The influence of antidepressants on the immune system. Arch Immunol Ther Exp (Warsz). 2019;67(3):143-151. doi:10.1007/s00005-019-00543-8

87. Di Rosso ME, Palumbo ML, Genaro AM. Immunomodulatory effects of fluoxetine: a new potential pharmacological action for a classic antidepressant drug? Pharmacol Res. 2016;109:101-107. doi:10. 1016/j.phrs.2015.11.021

88. Zumla A, Azhar EI, Arabi Y, et al. Host-directed therapies for improving poor treatment outcomes associated with the middle east respiratory syndrome coronavirus infections. Elsevier. 2015;71-74.

89. Yaseen AZEIA, Brian ABAMR, Eskild M, et al. Host-directed therapies for improving poor treatment outcomes associated with the middle east respiratory syndrome coronavirus infections. 2015.

90. Jiang HY, Deng M, Zhang YH, Chen HZ, Chen Q, Ruan B. Specific serotonin reuptake inhibitors prevent interferon- $\alpha$-induced depression in patients with hepatitis C: a meta-analysis. Clin Gastroenterol Hepatol. 2014;12(9):1452-60. e3. doi:10.1016/j.cgh.2013.04.035

91. Ris DR. COVID-19, interferons, and depression: a commentary. Psychiatry Res. 2020;113198.
92. Sommi RW, Crismon ML, Bowden CL. Fluoxetine: a serotonin-specific, second-generation antidepressant. Pharmacotherapy. 1987;7(1):1-15. doi:10.1002/j.1875-9114.1987.tb03496.x

93. Zuo J, Quinn KK, Kye S, Cooper P, Damoiseaux R, Krogstad P. Fluoxetine is a potent inhibitor of coxsackievirus replication. Antimicrob Agents Chemother. 2012;56(9):4838-4844. doi:10.1128/ AAC.00983-12

94. Peng L, Gu L, Li B, Hertz L. Fluoxetine and all other SSRIs are 5-HT2B agonists-importance for their therapeutic effects. Curr Neuropharmacol. 2014;12(4):365-379. doi:10.2174/ 1570159X12666140828221720

95. Stanley SA, Barczak AK, Silvis MR, et al. Identification of host-targeted small molecules that restrict intracellular Mycobacterium tuberculosis growth. PLoS Pathog. 2014;10(2): e1003946. doi:10.1371/journal.ppat.1003946

96. Ulferts R, van der Linden L, Thibaut HJ, et al. Selective serotonin reuptake inhibitor fluoxetine inhibits replication of human enteroviruses B and D by targeting viral protein 2C. Antimicrob Agents Chemother. 2013;57(4):1952-1956. doi:10.1128/AAC.02084-12

97. Organization WH. Mental health and psychosocial considerations during the COVID-19 outbreak, 18 March 2020. World Health Organization; 2020.

\section{Publish your work in this journal}

Therapeutics and Clinical Risk Management is an international, peerreviewed journal of clinical therapeutics and risk management, focusing on concise rapid reporting of clinical studies in all therapeutic areas, outcomes, safety, and programs for the effective, safe, and sustained use of medicines. This journal is indexed on PubMed Central, CAS,
EMBase, Scopus and the Elsevier Bibliographic databases. The manuscript management system is completely online and includes a very quick and fair peer-review system, which is all easy to use. Visit http://www.dovepress.com/testimonials.php to read real quotes from published authors. 